\title{
Expression of Androgen-Activating Enzymes in Cultured Cells of Developing Rat Brain
}

\author{
Angelo Poletti, Paola Negri-Cesi, Roberto Cosimo Melcangi, Alessandra Colciago, \\ Luciano Martini, and Fabio Celotti
}

Istituto di Endocrinologia, Università di Milano, Milano, Italy

\begin{abstract}
Dihydrotestosterone and estradiol, two active metabolites formed locally in the brain from testosterone, modulate several functions of the developing rat CNS; these compounds derive from the $5 \alpha$-reduction or the aromatization of the A-ring of the hormone. Also, progesterone and corticosteroids may be $5 \alpha$-reduced and subsequently $3 \alpha$-hydroxylated, becoming modulators of specific neuronal functions. Although the aromatase is a single enzyme, two types of $5 \alpha$-reductase have been cloned, showing peculiar biochemical properties and probably different functions. Therefore, the isoform(s) of the enzyme $5 \alpha$-reductase(s) present in early stage of brain development have been characterized in primary neuronal and glial cell cultures obtained from the fetal or neonatal rat brain, respectively. Aromatase expression was also studied. The results have shown that in all the brain cells examined type $15 \alpha$-reductase mRNA is expressed. No specific transcript of type $25 \alpha$-reductase is detectable in any of the cell types examined. Finally, the aromatase gene is expressed only in cultured fetal neurons and especially in those derived from the hypothalamic area of the rat embryos. It is interesting that no aromatase mRNA is detectable in mixed glia or in type 1 astrocytes and oligodendrocytes cultured separately. Key Words: Androgens - $5 \alpha$ - Reductases - Aromatase - Neurons Glial cells-Rat brain.

J. Neurochem. 68, 1298-1303 (1997).
\end{abstract}

Hormonal steroids are known to modulate several CNS functions. For instance, androgens appear to be responsible for the control of the sexual differentiation of the brain as well as of adult male sexual behavior; progesterone and deoxycorticosterone, in high doses, may exert anxiolytic and anesthetic properties (Paul and Purdy, 1992). It has been demonstrated that some of these actions may be mediated by steroid metabolites, rather than by the hormones themselves. The sexual differentiation of the brain is linked to the aromatization of testosterone to estrogens; the anxiolytic/ anesthetic properties of progesterone and of deoxycorticosterone are due to their respective $5 \alpha$-reduced- $3 \alpha$ hydroxylated metabolites, $3 \alpha$-hydroxy, $5 \alpha$-pregnan20-one (allopregnanolone or tetrahydroprogesterone), and 3 $\alpha, 21$-dihydroxy-5 $\alpha$-pregnan-20-one (tetrahydrodeoxycorticosterone) (Negri-Cesi et al., 1996). These compounds do not bind to classic steroid receptors but interact with the modulatory subunits of the GABAgated $\mathrm{Cl}^{-}$channel of the $\mathrm{GABA}_{\mathrm{A}}$ receptor (Majewska et al., 1986; Gee et al., 1988; Majewska, 1992). Therefore, it is presently believed that the enzymatic complexes responsible for these transformations, respectively, aromatase (Aro) and $5 \alpha$-reductase ( $5 \alpha-\mathrm{R}) 3 \alpha$ hydroxysteroid dehydrogenase system, play significant roles in regulating brain functions.

$5 \alpha-\mathrm{R}$ reduces the double bond in the $4-5$ position of the A-ring of several 3-keto- $\Delta^{4}$ steroids, e.g., testosterone, progesterone, deoxycorticosterone, corticosterone, etc., and is widely distributed in the rat brain (Celotti et al., 1992). 5 $\alpha$-R activity has been detected in several brain regions and structures and is particularly high in the white matter and in the myelin (Celotti et al., 1992). Moreover, among the various cell types present in the CNS, this enzymatic activity is much higher in neurons than in glial cells (oligodendrocytes and astrocytes) (Melcangi et al., 1993). Recently, two different isoforms of $5 \alpha-\mathrm{R}$ have been cloned (Russell and Wilson, 1994) and found to possess a limited degree of homology $(44 \%)$ as well as different biochemical properties: the $\mathrm{pH}$ optima are neutral to alkaline for the type 1 and acidic ( $\mathrm{pH} \mathrm{5.5)} \mathrm{for} \mathrm{the} \mathrm{type} 2$ isoform, and the affinity for the various substrates (testosterone, progesterone, corticosterone, etc.) is generally lower for the type 1 than for the type 2 isoform. Type 1 and type 2 isozymes also differ in their tissue and cellular distribution (Normington and Russell, 1992), in their subcellular compartmentalization (Poletti et al., 1996; Span et al., 1996), and in their sensitivity to inhibitors (Russell and Wilson, 1994). Because of

Received September 23, 1996; revised manuscript received November 6, 1996; accepted November 6, 1996.

Address correspondence and reprint requests to Dr. A. Poletti at Istituto di Endocrinologia, via Balzaretti 9, 20133 Milano, Italy.

Abbreviations used: Aro, aromatase; DHT, dihydrotestosterone; $3 \alpha$-diol, $5 \alpha$-androstane- $3 \alpha, 17 \beta$-diol; div, days in vitro; PCR, polymerase chain reaction; $5 \alpha-\mathrm{R}, 5 \alpha$-reductase; $\mathrm{RT}$, reverse transcription. 
these differences, it is expected that the two $5 \alpha$-R isozymes, even if they catalyze the same reaction, might exert different physiological functions. Moreover, it is interesting that the gene expression of the type $15 \alpha$ $\mathrm{R}$ is heterogeneous and may give rise to two forms, differing in four amino acids in the $\mathrm{N}$-terminal portion of the molecule; the two subforms of the $5 \alpha$-R type 1 possess different affinity for the substrates (LopezSolache et al., 1996).

Few data are available on the distribution of the two $5 \alpha-\mathrm{R}$ isozymes in the rat brain. Using in situ hybridization techniques, the presence of the mRNA for the type $15 \alpha-\mathrm{R}$ has been demonstrated in specific areas of the rat brain, with three distinct patterns of expression: during early fetal development type 1 mRNA is expressed in the proliferating region close to the ventricular wall of the CNS; during late fetal/early postnatal development, the expression in the ventricular zone decreases, whereas an increase of type $15 \alpha$-R expression is observed in the cortical plate and in the thalamus; and finally, in adult life, type $15 \alpha$-R expression appears to be confined to white matter structures (Lauber and Lichtensteiger, 1996). These results agree with the distribution of the activity of the enzyme found in the rat brain (Poletti et al., 1990; Celotti et al., 1992) and also with the distribution of the protein analyzed by immunohistochemistry (Tsuruo et al., 1996). It is important to note that another recent immunohistochemical study has shown the presence of the type 1 isozyme in glial cells, and not in neurons (Pelletier et al., 1994), in apparent conflict with the fact already mentioned that enzymatic activity is highest in neurons (Melcangi et al., 1993). This could suggest that the enzymatic activity found in neurons might be the type 2 isoform; however, the expression of the type $25 \alpha-\mathrm{R}$ in the rat brain is still controversial. Northern analysis performed on total RNA obtained from the whole brain of 7-week-old male rats has suggested that the CNS expresses both forms of the enzyme, with a great predominance of the type 1 isoform (Normington and Russell, 1992). More recently, using the same technique, the presence of only type $1 \mathrm{mRNA}$ has been described in the brain of male rats of the same age (Lephart, 1993); it must be recalled, however, that northern analysis is probably not sensitive enough to exclude the presence of type $2 \mathrm{mRNA}$ and consequently of the enzymatic protein.

The second androgen-activating enzyme, Aro, appears to be a single enzyme, with the same amino acid sequence in the different tissues in which it is present, even if its expression appears to be under the control of tissue-specific promoters. Studies on the regional distribution of Aro in the brain have indicated that this enzyme has a discrete distribution, particularly in the hypothalamus, the preoptic area, and the limbic system, i.e., in structures involved in the control of reproductive functions. Studies on the localization of the enzyme, performed by assaying the enzymatic activity in primary cultures of different brain cells (Negri-Cesi et al., 1992) and by immunohistochemical (Shinoda, 1994 ) or by in situ hybridization (Lauber and Lichtensteiger, 1994) techniques on the total rat brain, agree in indicating that only neurons aromatize androgens; however, the presence of a small amount of Aro or of its expression in other CNS cell types has not been carefully evaluated using the highly sensitive techniques presently available.

The aim of the present study was (a) to characterize the $\mathrm{pH}$ optima of the $5 \alpha-\mathrm{R}(\mathrm{s})$ present in primary cell cultures of neurons and mixed glia obtained from the fetal or neonatal rat brain, to obtain a possible indication on the enzyme isoform responsible for such conversion, and (b) to analyze the expression of the two types of $5 \alpha$-Rs and of Aro in the different cell types of the CNS, using the most powerful technique presently available, i.e., reverse transcription (RT) - polymerase chain reaction (PCR).

\section{MATERIALS AND METHODS}

\begin{abstract}
Animals
The embryos and the newborn rats were obtained from Sprague-Dawley mothers (Charles River, Italy) that were maintained in animal quarters with controlled temperature and humidity. The light schedule was $14 \mathrm{~h}$ of light and 10 $\mathrm{h}$ of dark (lights on at $0630 \mathrm{~h}$ ). The mothers were fed a standard pellet diet, and water was provided ad libitum.
\end{abstract}

\section{Cell cultures}

Neuronal cells were cultured from total brain of 15-dayold embryos obtained from time-pregnant mothers (day 0 $=$ sperm-positive smears) as previously described (Melcangi et al., 1993) and were used at 6 days in vitro (div); hypothalamic neuronal cell cultures were obtained from the hypothalamic region of the brain of 16-day-old embryos by dissolving the tissue in Dulbecco's modified Eagle's medium. The total cells in suspension were then plated for $1 \mathrm{~h}$ in $10-\mathrm{mm}$-diameter Petri dishes to remove fibroblast cells (which adhere rapidly to the plate), and the floating neurons were collected and plated on polylysine-coated 35-mm-diameter Petri dishes in phenol red-free Dulbecco's modified Eagle's medium containing 20\% fetal calf serum. After $24 \mathrm{~h}$ the medium was replaced with a chemically defined medium (Dulbecco's modified Eagle's medium without phenol red containing $50 \mathrm{U} / \mathrm{ml}$ penicillin, $50 \mathrm{U} / \mathrm{ml}$ streptomycin, $5 \mu \mathrm{g} /$ $\mathrm{ml}$ bovine insulin, $100 \mu \mathrm{g} / \mathrm{ml}$ bovine transferrin, $100 \mu M$ putrescine, and $20 \mathrm{n} M$ sodium selenite) until the collection of the cells ( 5 days div). Mixed glial cell cultures were obtained from 1- or 2-day-old newborn rats and were used at 19 div (Melcangi et al., 1993). Type 1 astrocyte cultures were obtained from mixed glial cultures (at 14 days div) by shaking the flasks overnight at $37^{\circ} \mathrm{C}$ and replating the cells from the bed layes according to the methods of McCarthy and DeVellis (1980); the astrocytes were used after 5 div. Oligodendrocyte cultures were obtained from primary cultures of mixed glia [5 div (Besnard et al., 1989)] and were used at $6 \operatorname{div}$ (Melcangi et al., 1993).

\section{Assay of the $5 \alpha-R$ activity}

To determine the pH optimum of the enzymatic isoform( $\mathrm{s}$ ) present in the cultured brain cells, the incubations were performed in phosphate-buffered saline solution (250 
$\mu 1$ ) at various $\mathrm{pH}$ values (ranging from 4 to 8 ) in the presence of an NADPH generating system (Poletti et al., 1990) and $3 \times 10^{-6} \mathrm{M}\left[{ }^{14} \mathrm{C}\right]$ testosterone (specific activity, $\sim 56.9$ $\mathrm{mCi} / \mathrm{mmol}$; Amersham, U.K.). The incubations were carried out for $2 \mathrm{~h}$ at $37^{\circ} \mathrm{C}$ in a Dubnoff metabolic shaker under a stream of $\mathrm{O}_{2} / \mathrm{CO}_{2}(98: 2)$. Vials without tissue provided the blanks for each $\mathrm{pH}$ value, and protein content was evaluated according to the method of Bradford (1976).

Detection of metabolites. At the end of the incubation the reaction was stopped by freezing the samples to $-20^{\circ} \mathrm{C}$. Tritium-labeled dihydrotestosterone (DHT) and $5 \alpha$-androstane- $3 \alpha, 17 \beta$-diol ( $3 \alpha$-diol) ( $\sim 5,000 \mathrm{dpm}$ each) were added to each sample to evaluate the recoveries. The metabolites formed were extracted twice with diethyl ether and separated by TLC using an eluting mixture of dichloromethane/diethyl ether $(11: 1 \mathrm{vol} / \mathrm{vol})$. The total $5 \alpha-\mathrm{R}$ activity was expressed as the sum of the two major testosterone metabolites, DHT and $3 \alpha$-diol.

\section{RT-PCR analysis}

Oligonucleotide sequences. All the synthetic oligonucleotides were deduced from published sequences of the cDNA of the two isoforms of $5 \alpha-R$ and of Aro and obtained from Pharmacia Biotech (Sweden). All the various types of brain cultured cells were solubilized in guanidium isothiocyanate, and total RNA was prepared by centrifugation over a $5.7 \mathrm{M}$ cesium chloride step gradient.

RT and PCR were performed using a GeneAmp kit (Perkin Elmer) on $2 \mu \mathrm{g}$ of total RNA from each sample. The same extracts of total RNA were subjected to specific analysis for the presence of $5 \alpha-\mathrm{R} 1,5 \alpha-\mathrm{R} 2$, and Aro mRNAs. Samples of total RNA obtained from both adult rat abdominal skin and prostate, known to contain high levels of both $5 \alpha-\mathrm{R} 1$ and $5 \alpha-\mathrm{R} 2$ mRNA (Russell and Wilson, 1994), were used as positive controls for $5 \alpha$-R isoforms; samples of total RNA extracted from adult rat ovary, known to contain high levels of Aro mRNA, or from rat placenta, which does not express the Aro gene, were used as positive or negative controls, respectively, for the Aro amplification. The synthetic oligonucleotides used as primers for the amplifications are schematically reported in Fig. 1, and the nucleotide sequences were as follows: for $5 \alpha-\mathrm{R} 1$ amplification, upstream primer, 5'-CGA CCT GCC TGG TTC ATA CA; downstream primer, 5'-GGT CAC CCA GTC TTC AGC AT; for $5 \alpha$-R 2 amplification, upstream primer, 5'-GTC CTG CTG GCT CTC TTC TC; downstream primer, $5^{\prime}$-CAG GCT TCC TGA GCT GGC GC; and for Aro amplification, upstream primer, 5'-TTG TTG TTA AAT ATG ATG CC; downstream primer, 5'-ATA CCA GGT CCT GGC TAC TG (Bulun et al., 1993). Genomic DNA amplification is avoided in the three types of analysis performed because of the properties of the set of primers chosen; those are located on different exons of each gene (as determined by comparison of the rat and human sequences alignments). Moreover, the RNA preparations were assayed in preliminary experiments in which the RT-PCR was performed omitting the addition of the reverse transcriptase in the samples. No specific bands were observed, indicating that genomic DNA could not be amplified.

The RT conditions were as follows: $42^{\circ} \mathrm{C}$ for $45 \mathrm{~min}$ followed by $5 \mathrm{~min}$ at $95^{\circ} \mathrm{C}$, using a final concentration of $1 \mathrm{~m} M$ each deoxynucleotide triphosphate, $1 \mathrm{U}$ of RNase inhibitor, $2.5 \mathrm{U}$ of murine leukemia virus reverse transcriptase, and the downstream primer in a final volume of $20 \mu \mathrm{l}$. The same buffer $(50 \mathrm{mM} \mathrm{KCl}, 10 \mathrm{~m} M$ Tris- $\mathrm{HCl}$, and $2 \mathrm{mMMgCl}$ ) was used for both transcription and amplification. The final amplification mixture comprised $2.5 \mathrm{U}$ of Ampli-Taq polymerase (GeneAmp Kit; Perkin-Elmer) and the upstream primer in a final volume of $100 \mu \mathrm{l}$. Samples were amplified by repeated cycles $(35)$ at $95^{\circ} \mathrm{C}$ for $1 \mathrm{~min}$, $42^{\circ} \mathrm{C}$ for $1 \mathrm{~min}$, and $72^{\circ} \mathrm{C}$ for $1 \mathrm{~min}$.

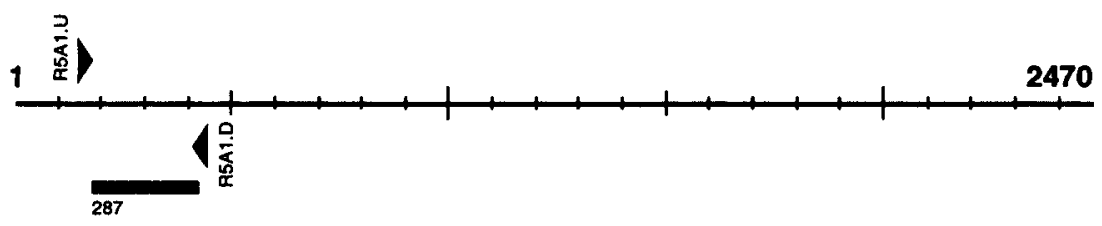

Type $15 \alpha-$ Reductase

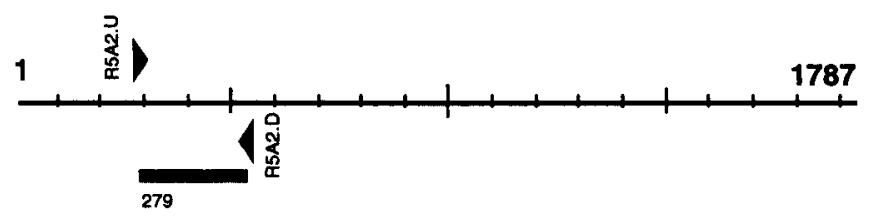

Type $25 \alpha-$ Reductase

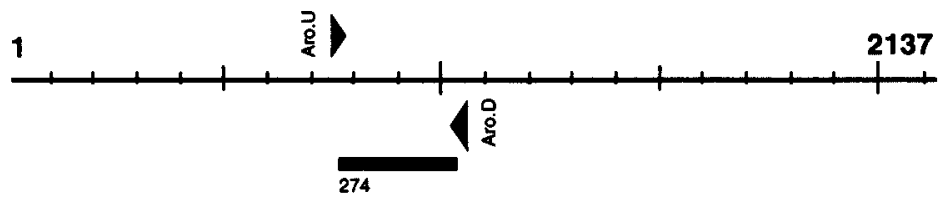

Aromatase

FIG. 1. Schematic representation of the positions of the three sets of oligoprimers, on the corresponding published cDNA, used in RT-PCR of the mRNAs of the two $5 \alpha-R$ isoforms and of Aro mRNAs. 
Amplification products were separated by electrophoresis in $2 \%$ agarose gel, which was visualized with ethidium bromide staining. The amplified cDNAs were transferred to a blotting membrane (Zeta-Probe; Bio-Rad, Richmond, CA, U.S.A.) by capillary elution in $10 \times \mathrm{SSC}$ (standard saline citrate) and fixed by baking at $80^{\circ} \mathrm{C}$ for $2 \mathrm{~h}$ under vacuum. The synthetic oligonucleotides used as radiolabeled probes in Southern analysis were as follows: $5 \alpha-\mathrm{R} 1$ oligoprobe, 5'-AGC AGA TAC TTG AGC CA; 5 $\alpha$-R 2 oligoprobe, 5'ACA TTA CTT CCA CAG GAC ATT T; and Aro oligoprobe, 5 '-CAC ATA ATG AAG CAC AAT CAT TA. The labeling reactions were performed on the free 5 ' end of the oligoprimers using T4 DNA polynucleotide kinase and $[\gamma-$ $\left.{ }^{32} \mathrm{P}\right] \mathrm{dATP}$. The cDNA on the membrane was then incubated at $45^{\circ} \mathrm{C}$ for $4 \mathrm{~h}$ with the prehybridizing solution, added to the $5^{\prime}-{ }^{32} \mathrm{P}$-end-labeled oligonucleotide probes $\left(1 \times 10^{6} \mathrm{cpm} /\right.$ $\mathrm{ml}$ ) specific for the cDNAs of the two isoforms of $5 \alpha-\mathrm{R}$ and of Aro, and hybridized at $45^{\circ} \mathrm{C}$ overnight. After washing the membranes were exposed to $\mathrm{x}$-ray films.

RT-PCR blanks were performed using distilled water and simultaneously subjected to RT-PCR-Southern blotting with the same reagents and conditions described. In any of the experiments performed no specific signal could be obtained, indicating that no contamination of any reagents occurred in these studies.

\section{RESULTS AND DISCUSSION}

This first set of experiments was designed to dissect out which $5 \alpha$-R isoform(s) are present in the rat brain, by taking advantage of the different $\mathrm{pH}$ optima of the two $5 \alpha-\mathrm{R}$ isoforms; the variation of the enzymatic activity of the $5 \alpha$-R has been analyzed as a function of the $\mathrm{pH}$ ( from 4 to 8 ) in lysates of cultured fetal neurons and neonatal mixed glia cells, by measuring the formation of labeled DHT and $3 \alpha$-diol from $\left[{ }^{14} \mathrm{C}\right]-$ testosterone. Figure 2 shows the pattern of $5 \alpha$-R activ-

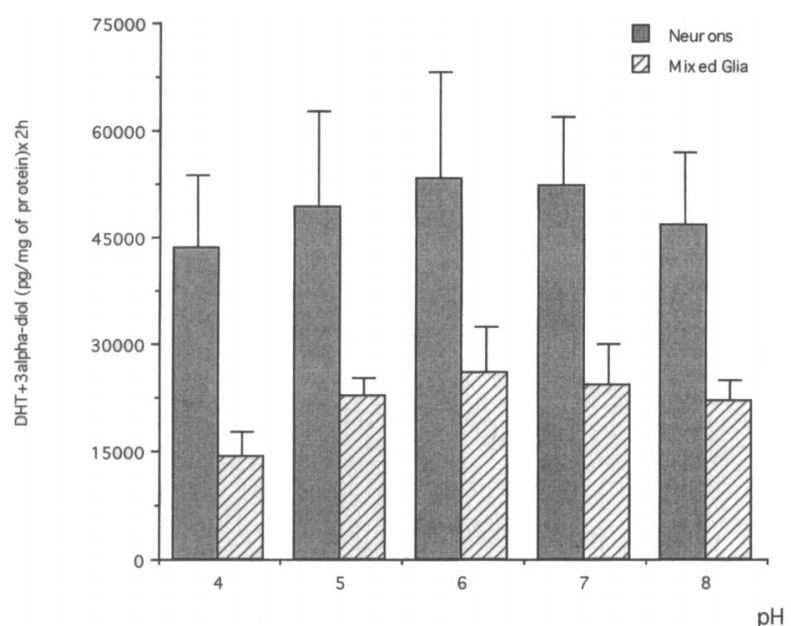

FIG. 2. Variation of the $5 \alpha-\mathrm{R}$ activity as a function of $\mathrm{pH}$ in cultured rat neurons derived from the brain of embryos at gestational day 15 and rat mixed glial cells derived from the brain of newborn animals. Enzymatic activity was measured as picograms per milligram of protein of labeled $5 \alpha$-reduced metabolites formed after incubation of $\left[{ }^{14} \mathrm{C}\right]$ testosterone, in the presence of NADPH as cofactor. See Materials and Methods for details.

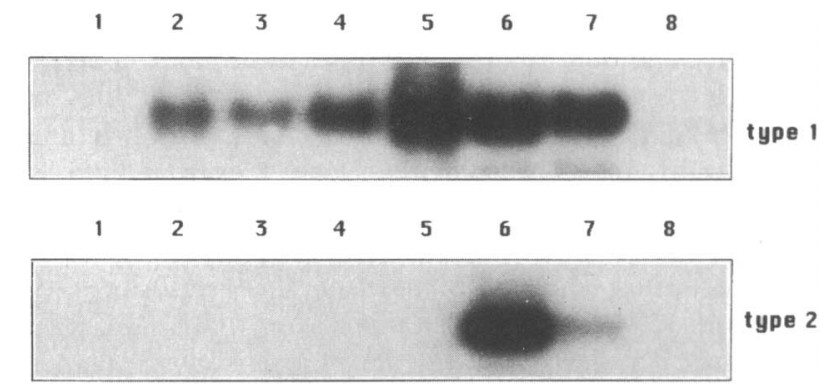

FIG. 3. Southern analysis performed on the RT-PCR product of amplification obtained using specific sets of oligoprimers for the two isoforms of the $5 \alpha$-R. The amplification was performed on the total RNA derived from the following samples: lane 1, DNA markers; lane 2, mixed glial cells; lane 3, type 1 astrocytes; lane 4 , oligodendrocytes; lane 5 , neurons derived from the whole brain; lane 6 , rat adult ventral prostate; lane 7 , rat abdominal skin; and lane 8, water.

ity in the two types of cell preparations, expressed as the sum of the amounts of the two $5 \alpha-\mathrm{R}$ metabolites formed per milligram of protein in $2 \mathrm{~h}$ of incubation. The results show that the $5 \alpha$-R activity present in rat fetal neurons obtained from total brain and in rat neonatal mixed glial cell cultures does not possess a clearcut $\mathrm{pH}$ dependency, being similarly active at $\mathrm{pH}$ values ranging from 4 to 8 ; no peak of activity was obtained for either type of cell culture at acidic $\mathrm{pH}$. Moreover, at all $\mathrm{pH}$ values, fetal neurons in culture possess about a twofold higher $5 \alpha-\mathrm{R}$ activity than mixed glial cell cultures obtained from newborn rats. These data seem to assign to the type 1 isoform the reducing activity present in the cells examined and confirm a previous finding indicating that neurons possess a $5 \alpha-\mathrm{R}$ activity higher than that of glial cells (Celotti et al., 1992; Melcangi et al., 1993). It is noteworthy that the characteristic peak of activity at $\mathrm{pH} 5.5$ of the $5 \alpha-\mathrm{R}$ type 2 is absent in both types of cultures; however, the enzymatic activity present at $\mathrm{pH} 4$ and 5 probably cannot be ascribed to the traditional type 1 alone because under these conditions, when the recombinant enzyme is expressed in mammalian cells (Normington and Russell, 1992) or in yeast cells (Poletti et al., 1996), type 1 activity is reduced to $50 \%$ or even more. One possibility to explain this activity is to postulate the existence in the rat brain of a subtype of the type 1 $5 \alpha$-R like the one recently described by Lopez-Solache et al. (1996), which is an elongated form (four amino acids in the $\mathrm{N}$-terminal region) derived from the same gene. Another possibility is the hypothesized existence of a third type of $5 \alpha-\mathrm{R}$ (Lephart, 1993), which, however, has not been cloned so far.

Figure 3 shows the autoradiography obtained after Southern analysis on the amplification products of the RT-PCR for type 1 or type $25 \alpha$-R performed on the RNAs of the different cultures of brain cells. A specific transcript of the type $15 \alpha$-R (upper panel) is detectable in RNA samples obtained from the mixed glia 
(lane 2), type 1 astrocytes (lane 3 ), oligodendrocytes (lane 4), and neurons (lane 5); the amplification products correspond in size to those observed in the rat prostate (lane 6) and in the rat abdominal skin (lane 7 ), used as positive controls. Lane 1 corresponds to the DNA markers, whereas lane 8 corresponds to the blank, in which no total RNA was added. No specific amplification products for the type 2 isoform (Fig. 3, lower panel) have been observed after the RT-PCR of the same samples tested for the type 1 isozyme (lanes 2-5), even after prolonged exposure of the membrane to $x$-ray film (data not shown). A specific band of hybridization of the expected size ( $279 \mathrm{bp}$ ) is amplified from the mRNAs of the two control tissues (lanes 6 and 7 ). These data indicate that, in the present experimental conditions, neither glial cells nor neurons are able to express the type $25 \alpha-\mathrm{R}$ gene. Even if RT-PCR is not a quantitative technique, it is interesting to note that the signal obtained in lane 5 (neurons) is almost two to three times higher (as evaluated by measuring the optic density of the corresponding spots; data not shown) than that obtained for glial cells, suggesting that, among brain cells, the neurons are those expressing the highest levels of type $15 \alpha$-R. These data confirm and extend those previously reported by this laboratory (Celotti et al., 1992; Melcangi et al., 1993), in which a much higher $5 \alpha$-R activity, as measured using a radioenzymatic procedure, was found in fetal neurons than in any type of neonatal glial cells. Pelletier et al. (1994) have reported results that apparently conflict with those described here. In fact, these authors failed to detect any $5 \alpha-\mathrm{R}$ immunoreactivity in the neuronal component of the rat brain using an antibody raised against the human type $15 \alpha-\mathrm{R}$; in their study, the material with the highest level of immunoreactivity was diffusely distributed throughout the cytoplasm of glial and ependymal cells. Possible explanations for this discrepancy may reside in several facts. First, the data presented here have been obtained by measuring the actual activity of the enzyme as well as its gene expression; in contrast, Pelletier et al. (1994) used only a morphological approach for "quantifying" the enzymatic protein. Moreover, the observations of Pelletier et al. (1994) were performed using an antibody raised against a synthetic peptide reproducing the sequence of the human type $15 \alpha-\mathrm{R}$, which is only partially conserved in the corresponding rat isoform (Normington and Russell, 1992; Pelletier et al., 1994); consequently, the possibility of a nonspecific reaction cannot be excluded, especially because the authors have not provided evidence on the efficiency of the antibody toward the rat isozyme.

The presence of the type $15 \alpha-\mathrm{R}$ gene expression and functional enzyme in all cells obtained from the fetal or perinatal rat CNS, and reported in the present study, agrees with a recent observation performed using in situ hybridization. In these studies the presence of the mRNA for this isoform in specific regions of the brain at different stages of development (Lauber and Lichtensteiger, 1996) has been analyzed. These data have shown that type $15 \alpha-\mathrm{R}$ mRNA is already expressed in early stages of fetal development (the first results were recorded on gestational day 12) and is maximal in the proliferating zones close to the ventricular wall. With the increase of the age of the animal, a substantially different localization was observed: the expression of the enzyme is especially elevated in the pyramidal cell layer of the hippocampus, in the subiculum, in the cortical plate, in the thalamus, and in the cerebellum at postnatal day 6 . It is interesting that at later intervals until adulthood, the level of the mRNA of the type 1 isoform is in contrast elevated in white matter structures, such as the optic chiasma, the corpus callosum, etc. Also, this result agrees with previous data of our laboratory (Poletti et al., 1990; Celotti et al., 1992). During the late (postnatal) stages of ontogeny, $5 \alpha$-R enzyme expression appears to parallel the process of myelinization (Celotti et al., 1992).

The expression of Aro in the different cell types of the brain was also investigated using RT-PCR. In these experiments, in addition to neurons of total fetal rat brain (gestational day 15), also those obtained from the hypothalamic region of the fetal rat brain (which are known to possess the highest Aro activity) at gestational day 16 were analyzed. Figure 4 shows the Southern analysis performed on the amplified products obtained with the RT-PCR technique. The rat ovary has been used as a positive control (lane 7), whereas the rat placenta, a tissue devoid of Aro mRNA, has been used as a negative control (lane 8). As shown in Fig. 4 , mixed glia (lane 2), type 1 astrocytes (lane 3 ), and oligodendrocytes (lane 4) do not contain any Aro transcripts. In contrast, in the mRNA samples of total brain neurons, and especially of hypothalamic neurons, a specific amplification product of the expected size was present in correspondence to that obtained from the rat ovary, indicating that the Aro gene is expressed only in neurons and is probably particularly expressed in those derived from the hypothalamus.

The specific localization of Aro in neurons and not
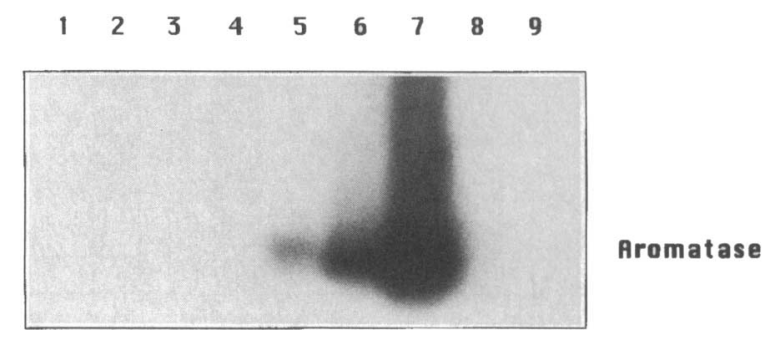

FIG. 4. Southern analysis performed on the RT-PCR product of amplification obtained using specific sets of oligoprimers for Aro. The amplification was performed on the total RNA derived from the following samples: lane 1, DNA markers; lane 2, mixed glial cells; lane 3, type 1 astrocytes; lane 4, oligodendrocytes; lane 5 , neurons derived from the whole brain; lane 6 , neurons derived from the hypothalamic area; lane 7 , rat ovary in estrus; lane 8 , rat placenta; and lane 9 , water. 
in glial cells has been previously described by immunohistochemical (Shinoda, 1994) and in situ hybridization (Lauber and Lichtensteiger, 1994) techniques and, in our laboratory, by a more direct approach, i.e., via the evaluation of the Aro activity in primary cultures of different brain cell populations (neuronal and glial cells) (Negri-Cesi et al., 1992). However, the Aro and its expression were recently found by in situ hybridization analysis, northern blot analysis, and measurement of the enzymatic activity in enriched glial cell cultures of the developing zebra finch telencephalon (Schlinger et al., 1994). It is obvious that a species difference may be responsible for this apparent discrepancy, as the zebra finch is a nonmammalian species.

Acknowledgment: This article is dedicated to the memory of Giuseppe Poletti. This work was supported by CNR funding (Special Projects ACRO grant 95.00395.PF39, FATMA grant 95.00868.PF41, and AGING grant 95.01020.PF40) by MURST and by AIRC.

\section{REFERENCES}

Besnard F., Perraud F., Sensenbrenner M., and Labourdette G. (1989) Effects of acid and basic fibroblast growth factors on proliferation and maturation of cultured rat oligodendrocytes. Int. J. Dev. Neurosci. Res. 21, 188-198.

Bradford M. M. (1976) A rapid and sensitive method for the quantitation of microgram quantities of protein utilizing the principle of protein-dye binding. Anal. Biochem. 72, 248-254.

Bulun S. E., Mahendroo M. S., and Simpson E. R. (1993) Polymerase chain reaction amplification fails to detect aromatase cytochrome P450 transcript in normal human endometrium or decidua. J. Clin. Endocrinol. Metab. 76, 1458-1463.

Celotti F., Melcangi R., and Martini L. (1992) The $5 \alpha$-reductase in the brain: molecular aspects and relation to brain function. Front. Neuroendocrinol. 13, 163-215.

Gee K. W., Bolger M. B., Brinton R. E., Coirini H., and McEwen B. S. (1988) Steroid modulation of the chloride ionophore in rat brain: structure-activity requirements, regional dependence and mechanism of action. J. Pharmacol. Exp. Ther. 246, 803812.

Lauber M. E. and Lichtensteiger W. (1994) Pre- and postnatal ontogeny of aromatase cytochrome P450 messenger ribonucleic acid expression in the male rat brain studied by in situ hybridization. Endocrinology 135, 1661-1668.

Lauber M. E. and Lichtensteiger W. (1996) Ontogeny of $5 \alpha$-reductase (type 1) messenger ribonucleic acid expression in rat brain: early presence in germinal zones. Endocrinology 137, 27182729.

Lephart E. D. (1993) Brain 5 $\alpha$-reductase: cellular, enzymatic, and molecular perspectives and implications for biological function. Mol. Cell. Neurosci. 4, 473-484.
Lopez-Solache I., Luu-The V., Séralini G.-E. and Labrie F. (1996) Heterogeneity of rat type $15 \alpha$-reductase cDNA: cloning, expression and regulation by pituitary implants and dihydrotestosterone. Biochim. Biophys. Acta 1305, 139-144.

Majewska M. D. (1992) Neurosteroids: endogenous bimodal modulation of the $\mathrm{GABA}_{\mathrm{A}}$ receptor. Mechanism of action and physiological significance. Prog. Neurobiol. 38, 379-395.

Majewska M. D., Harrison N. L., Schwartz R. D., Barker J. L., and Paul S. M. (1986) Steroid hormone metabolites are barbituratelike modulators of the GABA receptor. Science 232, $1004-$ 1007.

McCarthy K. D. and DeVellis J. (1980) Preparation of separate astroglial and oligodendroglial cell cultures from rat cerebral tissue. J. Cell Biol. 85, 890-902.

Melcangi R. C., Celotti F., Castano P., and Martini L. (1993) Differential localization of the $5 \alpha$-reductase and $3 \alpha$-hydroxysteroiddehydrogenase in neuronal and glial cultures. Endocrinology 132, $1252-1259$.

Negri-Cesi P., Melcangi R. C., Celotti F., and Martini L. (1992) Aromatase activity in cultured brain cells: difference between neurons and glia. Brain Res. 589, 327-332.

Negri-Cesi P., Poletti A., and Celotti F. (1996) Metabolism of steroids in the brain: a new insight in the role of $5 \alpha$-reductase and aromatase in the brain differentiation and functions. J. Steroid Biochem. Mol. Biol. 58, 455-466.

Normington K. and Russell D. W. (1992) Tissue distribution and kinetic characteristics of rat steroid $5 \alpha$-reductase isozymes. Evidence for distinct physiological functions. J. Biol. Chem. 267, $19548-19554$.

Paul S. M. and Purdy R. H. (1992) Neuroactive steroids. FASEB J. 6, 2311-2322.

Pelletier G., Luu-The V., and Labrie F. ( 1994) Immunocytochemical localization of $5 \alpha$-reductase in rat brain. Mol. Cell. Neurosci. 5, 394-399.

Poletti A., Celotti F., Melcangi R. C., Ballabio M., and Martini L. (1990) Kinetic properties of the $5 \alpha$-reductase of testosterone in the purified myelin, in the subcortical white matter and in the cerebral cortex of the male rat brain. J. Steroid Biochem. 35, 97-101.

Poletti A., Celotti F., Motta M., and Martini L. (1996) Characterisation of rat $5 \alpha$-reductases type 1 and type 2 expressed in yeast Saccharomyces cerevisiae. Biochem. J. 314, 1047-1052.

Russell D. W. and Wilson J. D. (1994) Steroid $5 \alpha$-reductase: two genes/two enzymes. Annu. Rev. Biochem. 63, 25-61.

Schlinger B. A., Amur-Umarjee S., Shen P., Campagnoni A. T, and Arnold A. P. (1994) Neuronal and non-neuronal aromatase in primary culture of developing Zebra-finch telencephalon. $J$. Neurosci. 14, 7541-7552.

Shinoda K. (1994) Brain aromatization and its associated structures. Endocr. J. 41, 115-138.

Span P. N., Sweep C. G. J., Benraad T. J., and Smals A. G. H. (1996) Differential subcellular distribution of rat prostatic steroid $5 \alpha$-reductase isozyme activities. Eur. J. Endocrinol. 134, 386-392.

Tsuruo Y., Miyamoto T., Yokoi H., Kitagawa K., Futaki S., and Ishimura K. (1996) Immunohistochemical presence of the $5 \alpha$ reductase rat type 1-containing cells in the rat brain. Brain Res. 722, 207-211 